\title{
INVESTIGATION OF CROSS FLOW FORCES INFLUENCE ON INCOMPRESSIBLE LAMINAR FLOW
}

\author{
M. N. Kogan ${ }^{1}$ and A. A. Basalaev ${ }^{2}$ \\ ${ }^{1}$ Central Aerohydrodynamic Institute (TsAGI) \\ Zhukovsky Str. 1, Zhukovsky, Moscow region 140180, Russia \\ ${ }^{2} \mathrm{MIPT}$ \\ Russia
}

The possibility to control different flows of gases is one of the most important in modern aerodynamics. The present investigation considers possibility to control gas flows using cross flow forces. There are several contradictory opinions about this problem. Herein, the influence of such forces on a duct flow is studied analytically and computationally. The results obtained show that cross flow forces may considerably change the flow in desired direction as well as in opposite one. Particularly, it is shown that the flow in the duct can be created by cross forces alone.

\section{PROBLEM DEFINITION}

The Navier-Stokes equations for incompressible stationary flow with presence of external source of momentum $\vec{F}(x, y, z)$ are as follows:

$$
\left.\begin{array}{c}
\rho\left(u \frac{\partial u}{\partial x}+v \frac{\partial u}{\partial y}+w \frac{\partial u}{\partial z}\right)=-\frac{\partial p}{\partial x}+\mu \Delta u+F_{x} ; \\
\rho\left(u \frac{\partial v}{\partial x}+v \frac{\partial v}{\partial y}+w \frac{\partial v}{\partial z}\right)=-\frac{\partial p}{\partial y}+\mu \Delta v+F_{y} ; \\
\rho\left(w \frac{\partial w}{\partial x}+v \frac{\partial w}{\partial y}+w \frac{\partial w}{\partial z}\right)=-\frac{\partial p}{\partial z}+\mu \Delta w+F_{z} ; \\
\frac{\partial u}{\partial x}+\frac{\partial v}{\partial y}+\frac{\partial w}{\partial z}=0 .
\end{array}\right\}
$$

This is an Open Access article distributed under the terms of the Creative Commons Attribution-Noncommercial License 3.0, which permits unrestricted use, distribution, and reproduction in any noncommercial medium, provided the original work is properly cited. 


\section{FLOW UNDER THE ACTION OF CROSS FORCES IN A LINEAR APPROXIMATION}

Equations (1) allow to get the following solution: $v=w=0, u=u(y)$.

Let consider small external momentum sources

$$
\vec{F}=\left\{0, F_{y}(x, y, z), F_{z}(x, y, z)\right\}
$$

that will cause small disturbances of pressure and velocity:

$$
\left.\begin{array}{c}
\vec{F}=\left\{0, F_{y}(x, y, z), F_{z}(x, y, z)\right\} ; \\
u=u_{0}+u_{1} ; \quad v=v_{1} ; \quad w=w_{1} ; \\
p=A x+p_{1}(x, y, z) .
\end{array}\right\}
$$

Substituting (2) in Eqs. (1) and disregarding the terms of the second order, finally, one will get:

$$
\left.\begin{array}{c}
\rho\left(u_{0} \frac{\partial u_{1}}{\partial x}+v_{1} \frac{\partial u_{0}}{\partial y}\right)=-\frac{\partial p_{1}}{\partial x}+\mu \Delta u_{1} ; \\
\rho u_{0} \frac{\partial v_{1}}{\partial x}=-\frac{\partial p_{1}}{\partial y}+\mu \Delta v_{1}+F_{y} ; \\
\rho u_{0} \frac{\partial w_{1}}{\partial x}=-\frac{\partial p_{1}}{\partial z}+\mu \Delta w_{1}+F_{z} ; \\
\frac{\partial u_{1}}{\partial x}+\frac{\partial v_{1}}{\partial y}+\frac{\partial w_{1}}{\partial z}=0 .
\end{array}\right\}
$$

In case of change of signs of forces $\left\{F_{y}, F_{z}\right\} \rightarrow\left\{-F_{y},-F_{z}\right\}$, to satisfy Eqs. (3), it will be necessary to change the signs of all other parameters. Thus, the dispute between scientists about the possibility of using cross flow forces only for drag reduction or only for drug increase is resolved.

\section{VERTICAL FORCES IN A DUCT}

Return now to nonlinear Eqs. (1).

First of all, let obtain expression for longitudinal velocity $u$ between two parallel plates (the coordinates of the plates are $y= \pm L / 2$ ) by the action of longitudinal gradient $A$ of the pressure without external disturbances (Fig. 1). 

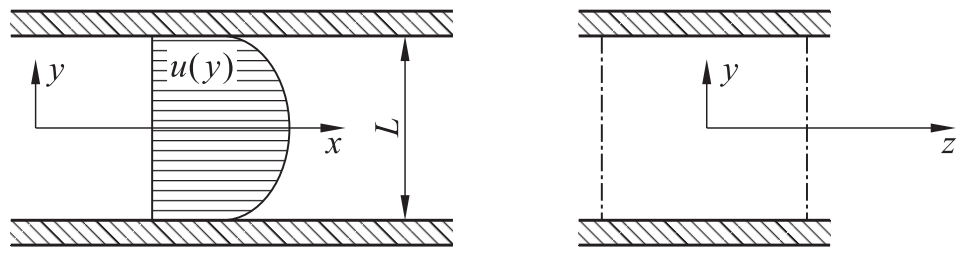

Figure 1 Geometry of a model used. Two parallel planes $y= \pm L / 2$ with inter space $L$ infinite in $x$ - and $z$-directions

For this flow, one can state that $\partial / \partial t=0, \partial / \partial x=0, u=u(y), u( \pm L / 2)$ $=0, v=w \equiv 0, p=A x$ and, substituting these in Eqs. (1), one will obtain $\mu\left(d^{2} u / d y^{2}\right)=A$, or

$$
u(y)=\frac{A}{2 \mu}\left(y^{2}-\left(\frac{L}{2}\right)^{2}\right) .
$$

The problem of the flow inside the duct has a precise solution for some sources of external forces. For example, consider $\vec{F}=\{0, B x y, 0\}$, i. e., vertical forces along the duct.

Navier-Stokes equations (1) allow the conditions where the flow velocity $u(y)$ depends on $y$ only: $v(x, y, z)=w(x, y, z)=0, \partial u / \partial x=0, u( \pm L / 2)=0$. Therefore, Eqs. (1) under the mentioned conditions take the following form:

$$
\frac{\partial p}{\partial x}=\mu \frac{\partial^{2} u}{\partial y^{2}} ; \quad \frac{\partial p}{\partial y}=B x y
$$

Thus, one has $p(x, y)=(B / 2) x y^{2}+C x$. Having it integrated under conditions $u( \pm L / 2)=0$, one finally gets:

$$
\begin{aligned}
u(y) & =\frac{B}{24 \mu}\left(y^{4}-\left(\frac{L}{2}\right)^{4}\right)+\frac{C}{2 \mu}\left(y^{2}-\left(\frac{L}{2}\right)^{2}\right) ; \\
\int_{-L / 2}^{L / 2} u(y) d y & =-\frac{B}{15}\left(\frac{L}{2}\right)^{5}-\frac{2 C}{3}\left(\frac{L}{2}\right)^{3} ; \\
\left.\mu \frac{d u}{d y}\right|_{w} & =-\frac{B}{6}\left(\frac{L}{2}\right)^{3}-C \frac{L}{2}
\end{aligned}
$$

Equation (4) is just a particular case of Eq. (6) with $C=A$ and $B=0$. Moreover, $C$ and $B$ can be changed independently creating new different flows that will satisfy Eqs. (5).

Both terms of Eq. (6) should be examined separately and the properties of the flows should be compared: 
1. It is obvious that $B=0$ leads to Poiseuille flow like Eq. (4) with $A=C$.

2. Cross forces alone (assuming $C=0$ ) can create longitudinal flow

$$
u(y)=\frac{B}{24 \mu}\left(y^{4}-\left(\frac{L}{2}\right)^{4}\right) .
$$

The direction will be positive when $B<0$.

3. Fixed gas consumption

$$
G=\int_{-L / 2}^{L / 2} u(y) d y
$$

can be produced by cross forces with $B=-15 G(2 / L)^{5}$ and with $C$ $=-(3 / 2) G(2 / L)^{3}$ separately. Corresponding wall frictions will be $\tau_{B}$ $=10 G / L^{2}$ and $\tau_{C}=6 G / L^{2}$. Consequently, the energy necessary to create the same flow through the canal is larger with cross flow forces.

Cross flow forces used above are just a particular case. For example, the similar results can be obtained with $F_{y}=B x y^{2 n-1}, n \in N$.

It is important to note that only in the presence of cross flow forces gradient in $x$ direction, one can obtain the changes in flow along $x$ axis.

\section{FLOW IN A DUCT UNDER ACTION OF THREE-DIMENSIONAL CROSS FORCES}

Consider the flow in a part of the canal which corresponds to the flow in the infinite canal in case of absence of forces.

Flow velocity $u$ (4) was set on the entrance of the duct and pressure at the outlet was fixed. Inside this short canal, cross flow forces were included.

\subsection{Boundary Conditions}

The number of the plane in Fig. 2 corresponds with the number of the boundary condition below:

1. Inlet $u(y)=u_{0}(y)$.

2. Symmetry plane: Normal velocity $v_{n}=0$, gradients of all other quantities in the normal direction $\partial / \partial \vec{n}=0$

3. Wall: $u=v=w=0$.

4. Boundary pressure: $p=$ fixed. 


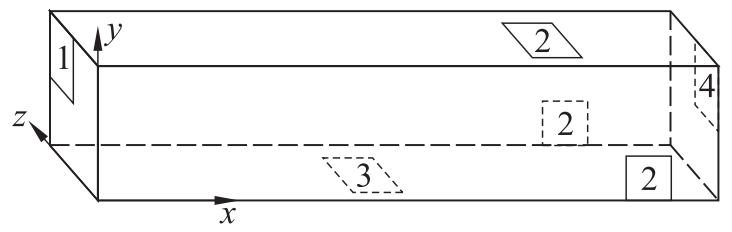

Figure 2 Computational domain. Modeling of the flow in an infinite duct

\subsection{Method Verification}

Method was verified on a flow without external forces, because it had an exact theoretical solution (4).

The pressure obtained on the inlet and the velocity profile on the outlet were compared with theoretical results.

Theory: $d p / d x=0.0241333$; calculation: $d p / d x=0.024125$; error $\sim 0.03 \%$.

Distribution of used cross flow forces is presented in Fig. 3. Due to the boundary conditions, the distribution of forces was symmetrical regarding to the planes $z=(b / 2) n, n \in N$, where $b / 2$ is the width of the computational zone. Force field was modulated by the function $M(x)=\sin (9(x-1)), 1 \leq x \leq 1.349$ in $x$ direction.

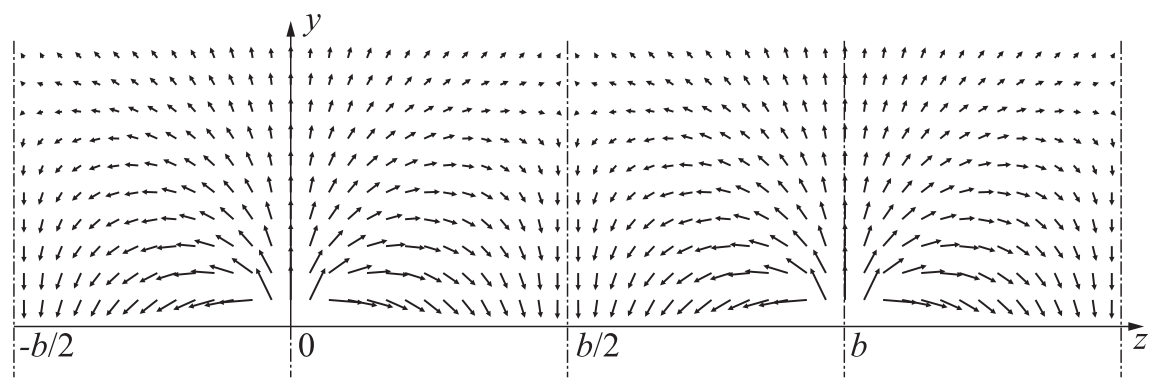

Figure 3 Distribution of cross flow forces obtained as an electrical density of infinite plane and set of tiny electrodes across of the plane

\subsection{Results}

Let consider modification of friction

$$
\bar{\tau}(x)=\left.\frac{2}{b} \int_{0}^{b / 2} \mu \frac{\partial u}{\partial y}\right|_{w} d z .
$$

Figure 4 shows relative change of value $\bar{\tau}(x) / \tau_{0}(x)$. 


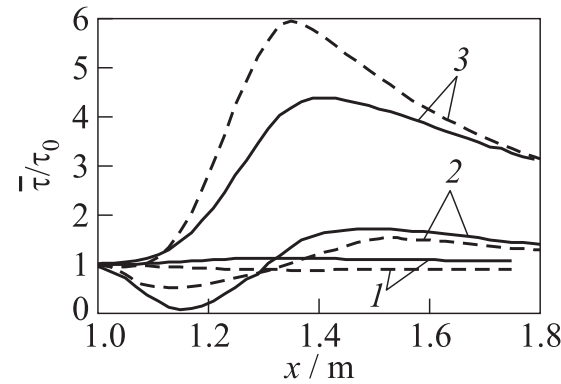

(a)

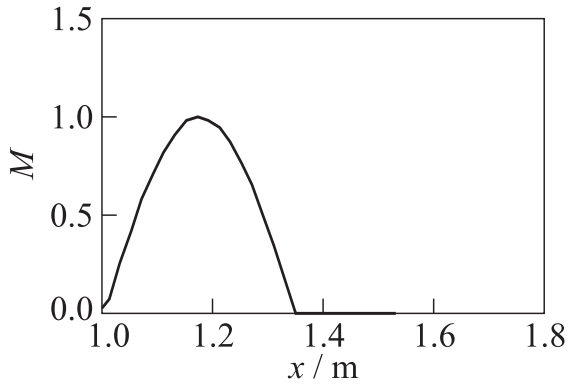

(b)

Figure 4 Relative change of $\bar{\tau}(x) / \tau_{0}(x): 1- \pm 0.01 M(x) ; 2- \pm 0.1 M(x) ; 3-$ $\pm M(x)$. Solid curves refer to positive meanings and dashed curves to negative

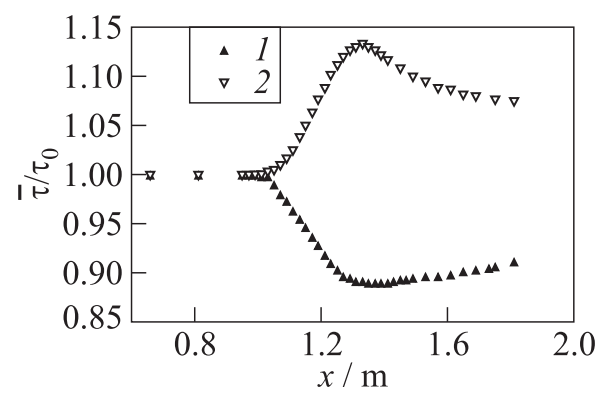

(a)

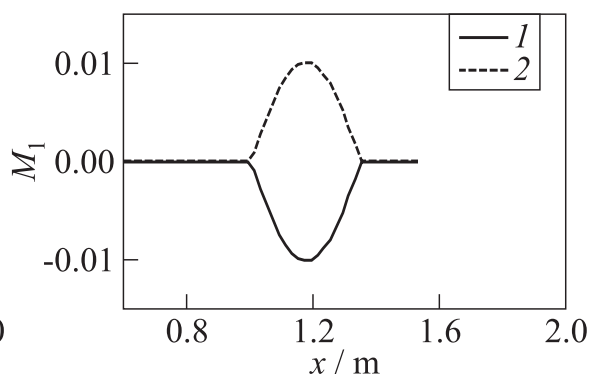

(b)

Figure 5 Sufficiently small opposite forces (1) result in opposite deviations of velocities, pressure, and, therefore, of shear stress $\bar{\tau}(x) / \tau_{0}(x)(2)$

Dependence of $\bar{\tau}(x)$ on $M(x)$ in very complex. High rates of forces cause an increase of friction all along the plate. However, some balance of parameters can lead to drag reduction. The tiniest considered forces $\pm 0.01 M(x)$ verify conclusion obtained in the beginning of the paper that small opposite forces result in opposite deviations of velocities and pressure (Fig. 5).

Distribution of local friction on the plate surface is shown in Fig. 6 .

The pressure on the inlet increased (Fig. 7) for the balance of total friction and momentum conservation to be obeyed.

\section{CONCLUDING REMARKS}

Careful consideration of Navier-Stokes equations shows that cross flow forces with longitudinal gradient can create a flow in a duct. 


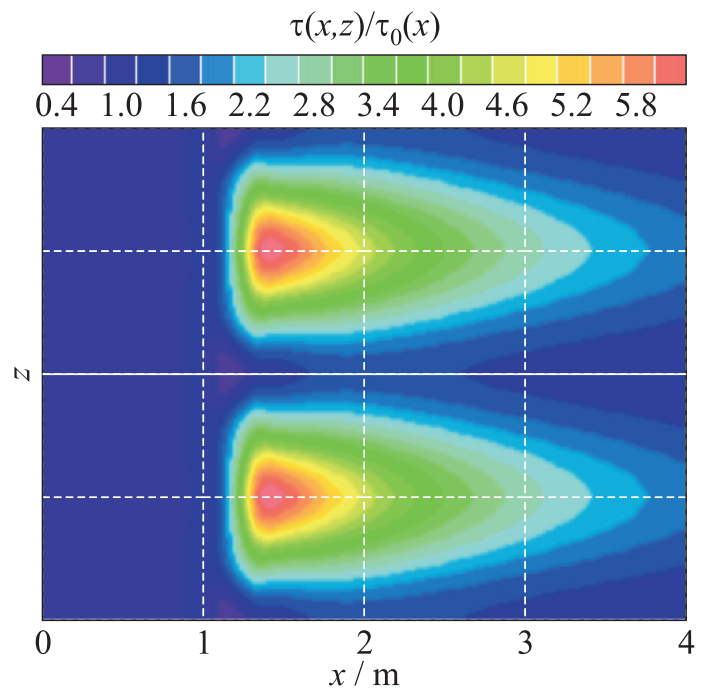

(a)

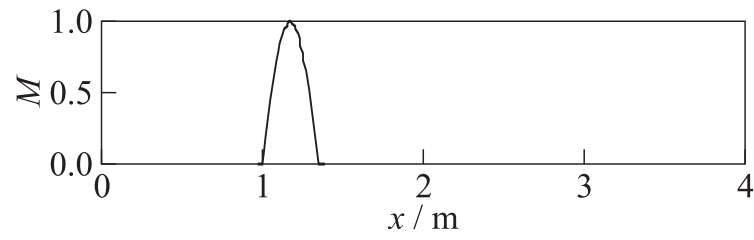

(b)

Figure 6 Distribution of relative local shear stress $\bar{\tau}(x, z) / \tau_{0}(x)(a)$ caused by the cross force $(b)$ on the plate surface. (Refer Kogan and Basalaev, p. 519.)

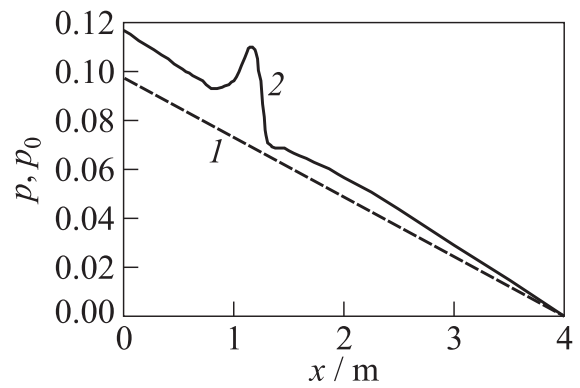

Figure 7 Cross-averaged pressure along $x$-axis; $p(x=4000)$ is forced to be zero by the boundary condition: 1 - Poiseuille flow pressure; and $2-3 \mathrm{D}$ flow with cross forces 
Computational part of investigation was made with rather general external momentum source which was dependent on $x, y$, and $z$. The influence of cross forces significantly changed the pressure and the friction on a plate. For the forces used, the increase of total drag of the plates was mostly obtained; however, the results showed the way to find the forces that can lead to the reduction of friction.

\section{ACKNOWLEDGMENTS}

The authors would like to thank the ISTC for financial support to postgraduate student Alexander Basalaev, and the EUCASS Programme Committee for granted opportunity to participate in EUCASS 2009. Also, we are very grateful to Mr. Courrech and Mr. Taran for their prompt help and useful advices. 